\title{
Two-Layer Observer Based Control for a Class of Uncertain Systems with Multi-Frequency Disturbances
}

\begin{abstract}
A novel disturbance estimation approach is presented for a class of uncertain systems subject to multiple-sinusoidal disturbances with unknown frequencies. Different from existing results on disturbance observer based control (DOBC), a new methodology with a two-layer observer structure is developed to effectively estimate and reject the disturbances. In the proposed control architecture, an auxiliary observer is derived to generate a disturbance representation in a parametric uncertainty form. Furthermore, the unknown parameters can be reduced to a constant vector with the dimension of the number of harmonic components in the disturbances. Then an augmented observer is designed to estimate the corresponding unknown parameters of the disturbances. As a result, the uncertain systems with disturbances constituting of multiple unknown-frequency sinusoidal components can be controlled within the DOBC framework, where asymptotic stability can be guaranteed. The proposed approach is successfully validated on a robotic manipulating example.
\end{abstract}

Keywords: Disturbance estimation and rejection, two layer observer, robotic manipulator, disturbance observer based control

\section{INTRODUCTION}

Over the past decades, the rejection problem of neutral stable disturbances for nonlinear systems has attracted considerable attentions. Among the many elegant approaches, the disturbance observer based control (DOBC), originated from frequency domain methods for linear systems, has been widely investigated with many successful applications ([1]-[7]).

Recently, DOBC has been developed for the cases with uncertain nonlinearities as well as more sophisticated disturbances. Representative results along this line of research include a robust DOBC approach developed in [8] for systems with nonlinearities, and a composite hierarchical antidisturbance control (CHADC) method can discussed in $([9,10$, 11]). The readers can be referred to [13]-[16] and references therein for some recent advances in this area. Note that most existing DOBC approaches for nonlinear systems requires the key information (such as frequency) of the disturbances $[3,7,8,9,10,11,12]$. It is still an open problem for DOBC design of uncertain nonlinear systems subject to disturbances with unknown frequencies and phases.

For other alternatives, such as output regulation methods and adaptive control approaches, the disturbances governed by exosystems with unknown parameters can be rejected in various situations. For linear systems, adaptive algorithms are proposed to handle multiple sinusoidal disturbances in [17, 18]. An adaptive unknown disturbance rejection scheme for nonlinear systems is provided in [19] by a combination of feedforward compensation and an adaptive identification technique.In [20], the authors consider the adaptive regulation problem for linear MIMO systems subject to unknown sinusoidal exogenous inputs. The problem is then extended to the cases of disturbances with the uncertain order of their exosystems [21]. The semiglobal output regulation problem is addressed in [22] for nonlin- ear systems with unknown parameters in the exosystem using adaptive internal model. Global stabilization and disturbance suppression are achieved in [23] via output feedback with a new formulation of internal model. Output regulation with unknown linear exosystems is discussed in [24] for uncertain nonlinear systems in the output feedback form, where the uncertain part is restricted to a particular form such that the adaptive technique can be designed to identify the unknown parameter. In [25], the uncertain nonlinear systems described in [24] are extended to some bounded function cases, such as polynomial functions.

Although output regulation methods and adaptive compensation approaches have been well deployed with many significant theoretical results and successful applications, it is still a challenging topic to develop a feasible anti-disturbance control framework for nonlinear systems with both harmonic disturbances and uncertainties. In our previous work [26], singlefrquency sinusoidal disturbances with unknown frequencies are estimated by constructing a generalized two-step observer for a class of multi-input-multi-output (MIMO )nonlinear system. For disturbances with a single frequency, the auxiliary observer can convert the problem to scalar estimation, which cannot be extended directly to the cases of multi-frequency disturbances. In the present paper, our primary motivation is to introduce a two-layer observer structure to excite and capture the disturbance characteristics, where the unknown signal can be represented in an observable form with appropriate coordinate transformation. In the presence of unmodeled dynamics, the proposed two-layer observer structure can be combined with feedback control design approaches to achieve desired control performance.

The rest of the paper is organized as follows. Section II describes the anti-disturbance control problem associated with a single-link robotic manipulator model. In Section 3, a two-layer 
disturbance observer is proposed, and a DOBC controller is designed for the composite systems with both disturbances and uncertainties in Section 4. In Section 5, the proposed method is applied to an example of a robotic manipulator, where numerical simulations demonstrate the advantages of the proposed scheme, followed by concluding remarks in Section 6 .

\section{PROBLEM FORMULATIONS}

We consider the anti-disturbance control problem of a singlelink robotic manipulator represented by the following model:

$$
M \ddot{q}+V_{m} \dot{q}+G(q)=\tau+d
$$

where $q \in R$ is the joint angular position, $\tau \in R$ is the torque applied to the joint, $M$ is bounded positive definite mass (or inertia) term, $V_{m}$ is viscous friction coefficient, and $G(q)$ is gravity term. We suppose that the joint angular position $q$ and the joint angular velocity $\dot{q}$ are measurable. The disturbance term $d$ in (1) is assumed to be a combination of multiple unknown sinusoidal signals represented as:

$$
\left\{\begin{array}{l}
d=d_{1}+d_{2}+\cdots+d_{m} \\
d_{i}=\Phi_{i} \sin \left(\omega_{i} t+\varphi_{i}\right)
\end{array}\right.
$$

where $\Phi_{i}, \omega_{i}, \varphi_{i}(i=1,2 \cdots m)$ are unknown constant parameters. Therefore the harmonic disturbance in the control input path is multi-sinusoidal signal with unknown amplitudes, phases and frequencies that can be also formulated by the following exogenous system

$$
\left\{\begin{array}{l}
\dot{w}=\Gamma w \\
d=V w
\end{array}\right.
$$

where $w \in R^{2 m}, \Gamma \in R^{2 m \times 2 m}$ has all its eigenvalues on the imaginary axis, and $V \in R^{1 \times 2 m}$ is proper unknown matrix. For simplicity, we denote

$$
\Gamma=\left[\begin{array}{lll}
\Gamma_{1} & 0 & 0 \\
0 & \ddots & 0 \\
0 & 0 & \Gamma_{m}
\end{array}\right], V=\left[\begin{array}{llll}
V_{1} & V_{2} & \cdots & V_{m}
\end{array}\right]
$$

and without loss of generality, suppose that $\left[\Gamma_{i}, V_{i}\right](i=$ $1,2 \ldots m$ ) has observable canonical form described as

$$
\Gamma_{i}=\left[\begin{array}{ll}
0 & 1 \\
-\omega_{i}^{2} & 0
\end{array}\right], V_{i}=\left[\begin{array}{ll}
1 & 0
\end{array}\right]
$$

In this paper we would like to address the problem of rejecting multi-sinusoidal disturbances of unknown parameters for nonlinear uncertain systems. In particular, we consider the uncertainty of (1) obeying

$$
h(\dot{q}, q):=V_{m} \dot{q}+G(q)=h_{1}(\dot{q}, q)-\Delta h(\dot{q}, q),
$$

where $h_{1}(\dot{q}, q)$ and $\Delta h(\dot{q}, q)$ represent known and uncertainty parts in $h(\dot{q}, q)$ respectively. For any pair of $\left[\begin{array}{c}q \\ \dot{q}\end{array}\right]$ and $\left[\begin{array}{c}\bar{q} \\ \dot{\bar{q}}\end{array}\right]$, the nonlinear uncertain term is supposed to satisfy

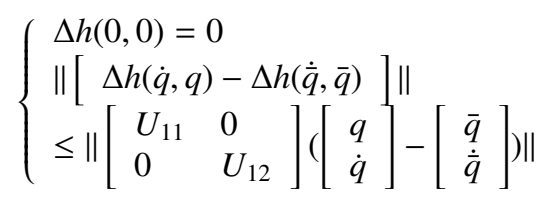

where $U_{11}$ and $U_{12}$ are given constant weighting matrix. Note that the model discussed in the present paper can be easily extended to more general nonlinear systems for anti-disturbance control.

\section{TWO LAYER DISTURBANCE OBSERVER DESIGN}

The block diagram of proposed strategy can be shown in Fig.1, where the nominal feedback controller is designed to guarantee stability of the closed loop system in the absence of disturbances. To deal with disturbances with multiple unknown sinusoidal components, a two-layer disturbance observer is introduced constituting of an auxiliary observer and an augmented observer structure. The auxiliary observer yields a disturbance presentation in a parametric uncertainty form, and the augmented observer is constructed to obtain the unknown parameters related to multi-sinusoidal disturbances, with which the disturbance generator provides an estimate of the disturbances according to the relations between $\xi, \theta_{o}$ and $d$.

\subsection{AUXILIARY DISTURBANCE OBSERVER}

We first recall some recent results in the literature ([3, 8, 9, 7]), and notice that the disturbance observer can not be constructed directly if the information of disturbance frequencies is not available. For the case of single-frequency disturbances, a possible solution, as discussed in [26], can be derived based on a representation of the disturbance by a nonlinear function related to an unknown scalar. The situation of those with multifrequency disturbances is more complicated because the unknown parameters are now in a vector form, which prevents us from designing disturbance observers based on existing results. In this paper, a novel two layer observer structure is explored to estimate disturbances with multiple unknown frequency components. We start with the following result on a representation of disturbance $d(t)$.

Theorem 1: Consider system

$$
\dot{\dot{\xi}}(t)=\breve{G} \breve{\xi}(t)+\breve{L} d
$$

where the multi-sinusoidal disturbance $d$ is described as in (2). If Hurwitz matrix $\breve{G} \in R^{2 m \times 2 m}$ and $\breve{L} \in R^{2 m \times 2 m}$ satisfy (10) then the multi-sinusoidal signal can be expressed by

$$
d(t)=\breve{\Xi}_{o}(t) \breve{\theta}_{o}+\breve{\Xi}_{e}(t) \breve{g}_{e}+\breve{\theta}^{T} \breve{\delta}(t)
$$

where

$$
\begin{array}{r}
\breve{\Xi}_{o}(t)=\left[\begin{array}{llll}
\breve{\xi}_{1}(t) & \breve{\xi}_{3}(t) & \cdots & \breve{\xi}_{2 m-1}(t)
\end{array}\right], \\
\breve{\Xi}_{e}(t)=\left[\begin{array}{llll}
\breve{\xi}_{2}(t) & \breve{\xi}_{4}(t) & \cdots & \breve{\xi}_{2 m}(t)
\end{array}\right]
\end{array}
$$




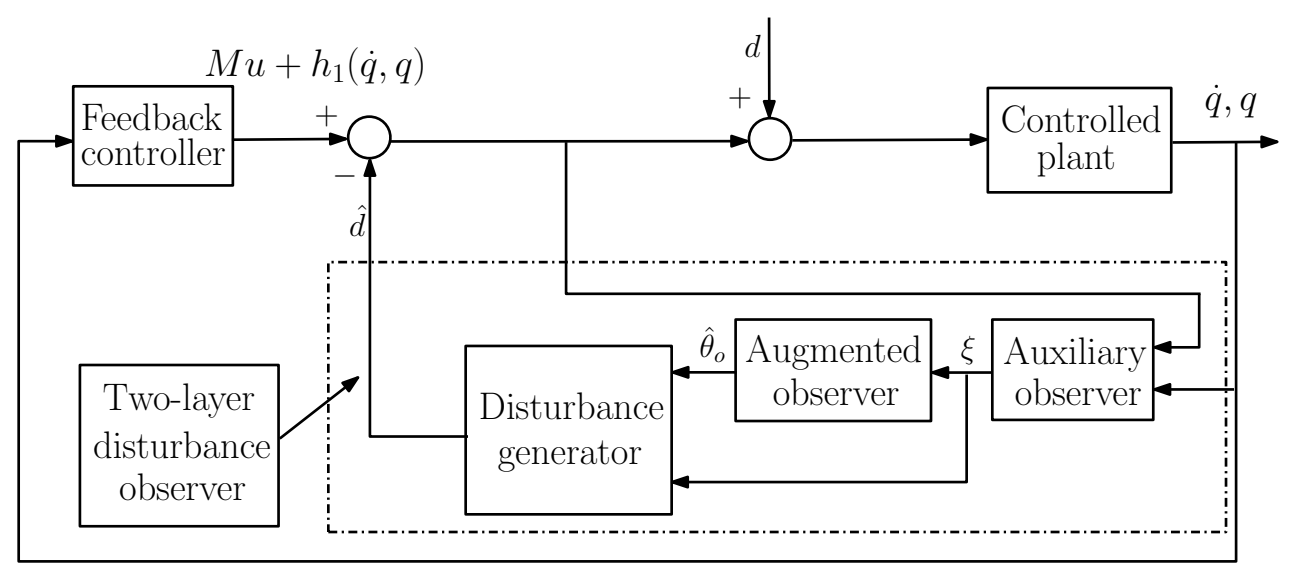

Figure 1: Block Diagram of the proposed control architecture

$$
\begin{gathered}
\breve{\xi}(t)=\left[\begin{array}{c}
\breve{\xi}_{1}(t) \\
\breve{\xi}_{2}(t) \\
\vdots \\
\breve{\xi}_{2 m}(t)
\end{array}\right], \breve{g}_{e}=\left[\begin{array}{c}
\breve{g}_{2} \\
\breve{g}_{4} \\
\vdots \\
\breve{g}_{2 m}
\end{array}\right], \\
\breve{\theta}_{o}=\left[\begin{array}{c}
\breve{\theta}_{1} \\
\breve{\theta}_{3} \\
\vdots \\
\breve{\theta}_{2 m-1}
\end{array}\right], \breve{\theta}=\left[\begin{array}{c}
\breve{\theta}_{1} \\
\breve{\theta}_{2} \\
\vdots \\
\breve{\theta}_{2 m}
\end{array}\right],
\end{gathered}
$$

with $\breve{\theta}_{o}, \breve{\theta}$ being unknown vectors of appropriate dimensions and $\breve{\delta}(t)$ satisfying

$$
\dot{\breve{\delta}}(t)=\breve{G} \breve{\delta}(t),
$$

where

$$
\breve{G}=\left[\begin{array}{cccc}
0 & 1 & \cdots & 0 \\
\vdots & \vdots & \vdots & \vdots \\
0 & 0 & \cdots & 1 \\
-\breve{g}_{1} & -\breve{g}_{2} & \cdots & -\breve{g}_{2 m}
\end{array}\right], \breve{L}=\left[\begin{array}{c}
0 \\
0 \\
\vdots \\
1
\end{array}\right] .
$$

Proof: By virtue of the lemma in [19], we can conclude that the harmonic disturbance $d(t)$ in (3) can be presented in the form of

$$
d(t)=\breve{\xi}^{T}(t) \breve{\theta}+\breve{\theta}^{T} \breve{\delta}(t)
$$

with

$$
\dot{\check{\delta}}(t)=\breve{G} \breve{\delta}(t),
$$

where $\breve{\theta}$ is an unknown vector related to disturbance frequencies. Applying (11) into (7) yields

$$
\dot{\dot{\xi}}=\bar{G} \breve{\xi}+\breve{L} \breve{\theta} T \breve{\delta}(t)
$$

where

$$
\bar{G}=\left[\begin{array}{cccc}
0 & 1 & \cdots & 0 \\
\vdots & \vdots & \vdots & \vdots \\
0 & 0 & \cdots & 1 \\
-\breve{g}_{1}+\breve{\theta}_{1} & -\breve{g}_{2}+\breve{\theta}_{2} & \cdots & -\breve{g}_{2 m}+\breve{\theta}_{2 m}
\end{array}\right]
$$

As $\breve{\delta}(t)$ is a decaying vector, the steady property of $\breve{\xi}$ depends on matrix $\bar{G}$. From (8) we can see that $\breve{\xi}$ is an output of a lowpass filter input by a harmonic signal,which will converge to another harmonic signal with the same frequencies as $d$. Thus the polynomial of $\bar{G}$ is equal to that of $\Gamma$,i.e.

$$
\begin{gathered}
\left(s^{2}+\omega_{1}^{2}\right)\left(s^{2}+\omega_{2}^{2}\right)+\cdots+\left(s^{2}+\omega_{m}^{2}\right) \\
=s^{2 m}+\left(\breve{g}_{2 m}-\breve{\theta}_{2 m}\right) s^{2 m-1}+\cdots+\left(\breve{g}_{1}-\breve{\theta}_{1}\right)
\end{gathered}
$$

With this, it is straightforward that for $i=1,2 \cdots m$

$$
\breve{\theta}_{2 i}=\breve{g}_{2 i},
$$

Then Equ.(8) can be obtained by combining (11) with (12), which completes the proof.

Based on the representation of the disturbance in (8), we are now ready to derive the correlation between unknown frequencies and the proposed auxiliary observer. Consider a class of nonlinear affine for system (1)

$$
\begin{aligned}
& \xi=v+\psi, \psi=L M \dot{q} \\
& \dot{v}=G(v+\psi)+L h_{1}(\dot{q}, q)-L \tau,
\end{aligned}
$$

where $G \in R^{2 m \times 2 m}$ is Hurwitz, which is a free parameter to be determined together with $L$. We assume that $(G, L)$ is controllable, and vector $\xi, v, \psi$ have compatible dimensions. $G, L$ and $\xi$ satisfy

$$
\xi=\left[\begin{array}{c}
\xi_{1} \\
\xi_{2} \\
\vdots \\
\xi_{m}
\end{array}\right], \xi_{i}=\left[\begin{array}{c}
\xi_{i, 1} \\
\vdots \\
\xi_{i, m}
\end{array}\right]
$$

$$
G=\left[\begin{array}{ccc}
G_{1} & 0 & 0 \\
0 & \ddots & 0 \\
0 & 0 & G_{m}
\end{array}\right], L=\left[\begin{array}{c}
L_{1} \\
L_{2} \\
\vdots \\
L_{m}
\end{array}\right]
$$




$$
G_{i}=\left[\begin{array}{cccc}
0 & 1 & \cdots & 0 \\
\vdots & \vdots & \vdots & \vdots \\
0 & 0 & \cdots & 1 \\
-g_{i, 1} & -g_{i, 2} & \cdots & -g_{i, 2 m}
\end{array}\right], L_{i}=\left[\begin{array}{c}
0 \\
0 \\
\vdots \\
1
\end{array}\right]
$$

where $g_{i, j}, i=1,2 \cdots m, j=1,2 \cdots 2 m$ are constant values which can guarantee Hurwitz matrix of $G$. Recall (1) and (13). We have

$$
\dot{\xi}=G \xi+L d+L \Delta h(\dot{q}, q) .
$$

Lemma 2: If there exists vector-functions $\xi_{i}$ in (14), satisfying

$$
\dot{\xi}_{i}(t)=G_{i} \xi_{i}(t)+L_{i}(d+\Delta h(\dot{q}, q)
$$

then the disturbance $d(t)$ in (3) can be presented in the form of

$$
d(t)=\Xi_{i o}(t) \theta_{i o}+\Xi_{i e}(t) g_{i, e}+\theta_{i}^{T} \delta_{i}(t)
$$

where

$$
\begin{aligned}
& \Xi_{i o}(t)=\left[\begin{array}{llll}
\xi_{i, 1}(t) & \xi_{i, 3}(t) & \cdots & \xi_{i,(2 m-1)}(t)
\end{array}\right], \\
& \Xi_{i e}(t)=\left[\begin{array}{lll}
\xi_{i, 2}(t) & \xi_{i, 4}(t) \cdots & \xi_{i, 2 m}(t)
\end{array}\right], \\
& \theta_{i}=\left[\begin{array}{c}
\theta_{i, 1} \\
\theta_{i, 2} \\
\vdots \\
\theta_{i, m}
\end{array}\right], \theta_{i o}=\left[\begin{array}{c}
\theta_{i, 1} \\
\theta_{i, 3} \\
\vdots \\
\theta_{i,(2 m-1)}
\end{array}\right], g_{i, e}=\left[\begin{array}{c}
g_{i, 2} \\
g_{i, 4} \\
\vdots \\
g_{i, 2 m}
\end{array}\right]
\end{aligned}
$$

where $\theta_{i}$ is an unknown constant vector to be determined and $\delta_{i}$ obeys

$$
\dot{\delta}_{i}(t)=G_{i} \delta_{i}(t)-L_{i} \Delta h(\dot{q}, q)
$$

Proof: Based on Theorem 1, there exists a constant vector $\hat{\theta}=\theta_{i}$ such that $d$ can be expressed as

$$
\begin{aligned}
d(t) & =\hat{\xi}^{T} \theta_{i}+\theta_{i}^{T} \hat{\delta} \\
& =\left(\hat{\xi}-\xi_{i}+\xi_{i}\right) \theta_{i}+\theta_{i}^{T} \hat{\delta}_{i}=\xi_{i}^{T} \theta_{i}+\theta_{i}^{T} \hat{\delta}+\theta_{i}^{T}\left(\hat{\xi}-\xi_{i}\right)
\end{aligned}
$$

According to (7),(9) and (18) it follows that

$$
\begin{aligned}
\dot{\hat{\xi}}-\dot{\xi}_{i} & =G\left(\hat{\xi}-\xi_{i}\right)-L_{i} \Delta h(\dot{q}, q), \dot{\hat{\xi}}-\dot{\xi}_{i}+\dot{\hat{\delta}} \\
& =G\left(\hat{\xi}-\xi_{i}+\hat{\delta}\right)-L_{i} \Delta h(\dot{q}, q) .
\end{aligned}
$$

Recall Theorem 1 and the equations of (20) and (23). It can be seen that

$$
d(t)=\xi_{i}^{T} \theta_{i}+\theta_{i}^{T} \delta_{i}(t)=\Xi_{i o}(t) \theta_{i o}+\Xi_{i e}(t) g_{i e}+\theta_{i}^{T} \delta_{i}(t)
$$

where $\delta_{i}(t)=\hat{\xi}-\xi_{i}+\hat{\delta}$ satisfying (21). Thus the proof is completed.

Q.E.D

\subsection{AUGMENTED DISTURBANCE OBSERVER}

In this section, the unknown constant vector $\theta_{i o}$ in (19) will be estimated by a novel method with a fast convergence property different from adaptive algorithms. Before estimating the disturbance based on auxiliary observer (13), we would like to investigate the problem with more analysis. From (8) and (11) we have

$$
\left\{\begin{array}{l}
\dot{\xi}_{i}(t)=G_{i} \xi_{i}(t)+L_{i}\left(\Xi_{i o}(t) \theta_{i o}+\Xi_{i e}(t) g_{i e}(t)\right. \\
\left.+\Delta h(\dot{q}, q)+\theta_{i}^{T} \delta_{i}(t)\right) \\
\dot{\xi}_{i, 2 m}(t)=-\Xi_{i o}(t) g_{i o}+\Xi_{i o}(t) \theta_{i o}+\Delta h(\dot{q}, q)+\theta_{i}^{T} \delta_{i}(t)
\end{array}\right.
$$

where

$$
g_{i e}=\left[\begin{array}{c}
g_{i, 2} \\
\vdots \\
g_{i, 2 m}
\end{array}\right], \theta_{i o}=\left[\begin{array}{c}
\theta_{i, 1} \\
\vdots \\
\theta_{i, 2 m-1}
\end{array}\right], g_{i o}=\left[\begin{array}{c}
g_{i, 1} \\
\vdots \\
g_{i, 2 m-1}
\end{array}\right] .
$$

In (24), the nonlinear term $\Xi_{i o}(t) \theta_{i o}$ is not in an observable for$\mathrm{m}$, which is not convenient for constructing an observer to estimate $\theta_{i o}$ directly. From (12) we can see that unknown vector $\theta_{i o}$ is a polynomial function of $\omega_{i}, g_{i o}, i=1,2 \cdots m$, i.e. $\theta_{i o}$ is determined by $g_{i o}$ with a given disturbance $d(t)$. If we set $g_{1 o}=g_{2 o}=\cdots g_{m o}=g_{o}$, we have $\theta_{1 o}=\theta_{2 o}=\cdots \theta_{m o}=\theta_{o}$.

Repeating the above process (24),we get the following augmented equation

$$
\dot{\bar{\xi}}_{2 m}(t)=-\bar{\Xi}_{o}(t) g_{o}+\bar{\Xi}_{o}(t) \theta_{o}+\hat{L} \Delta h(q, q)+\theta^{T} \delta(t)
$$

where

$$
\begin{aligned}
& \bar{\xi}_{2 m}(t)=\left[\begin{array}{c}
\xi_{1,2 m}(t) \\
\xi_{2,2 m}(t) \\
\vdots \\
\xi_{m, 2 m}(t)
\end{array}\right], \bar{\Xi}_{o}(t)=\left[\begin{array}{c}
\Xi_{1 o}(t) \\
\Xi_{2, o}(t) \\
\vdots \\
\Xi_{m, o}(t)
\end{array}\right], \\
& \left.\delta_{(} t\right)=\left[\begin{array}{c}
\delta_{1}(t) \\
\delta_{2}(t) \\
\vdots \\
\delta_{m}(t)
\end{array}\right], \theta=\operatorname{diag}\left\{\theta_{1}, \theta_{2} \cdots \theta_{m}\right\}, \\
& \hat{L}=\left[\begin{array}{llll}
1 & 1 & \cdots & 1
\end{array}\right]^{T}, \hat{L} \in R^{m \times 1}
\end{aligned}
$$

The dimension of $\Xi_{o}$ inspires us to solve this problem from the perspective of observer design. Thus an augmented observer structure is designed as follows

$$
\begin{aligned}
\hat{\theta}_{o}= & z+p \\
\dot{z}= & -\alpha \bar{\Xi}_{o}^{\prime}(t) \bar{\Xi}_{o}(t) \hat{\theta}_{o}(t)+\alpha \bar{\Xi}_{o}^{\prime}(t) \bar{\Xi}_{o}(t) g_{o} \\
& -\alpha \bar{\Xi}_{e}^{\prime}(t) \bar{\xi}_{2 m}(t) \\
p= & \alpha \bar{\Xi}_{o}^{\prime}(t) \bar{\xi}_{2 m}(t)
\end{aligned}
$$

where

$$
\bar{\Xi}_{e}(t)=\left[\begin{array}{c}
\Xi_{1 e}(t) \\
\Xi_{2 e}(t) \\
\vdots \\
\Xi_{m e}(t)
\end{array}\right]
$$

and $\alpha>0$ is a constant value to be determined. By virtue of (17) we get

$$
\dot{\bar{\Xi}}_{o}(t)=\bar{\Xi}_{e}(t) .
$$


Then we obtain the following error model by differentiating estimation error $\tilde{\theta}_{o}=\theta_{o}-\hat{\theta}_{o}$ in view of (27) and (28),

$$
\begin{aligned}
\dot{\tilde{\theta}}_{o} & =-\alpha \Xi_{o}^{2} \tilde{\theta}_{o}-\alpha \Xi_{o} \theta^{T} \delta(t)-\alpha \Xi_{o} \hat{L} \Delta h(\dot{q}, q), \\
\dot{\delta}(t) & =G \delta(t)-L \Delta h(\dot{q}, q)
\end{aligned}
$$

When $\Delta h(\dot{q}, q)$ in system model (1) is considered, the dynamic property of (29) turns out to be complicated. However an advantage of the proposed DOBC framework is that $\Delta h(\dot{q}, q)$ doesn't need to be identified as traditional adaptive algorithms. Instead, $\Delta h(\dot{q}, q)$ and $d$ will be attenuated and rejected simultaneously by combining the observer design with a robust controller, which will be shown in the next section.

\section{DOBC WITH STABILITY ANALYSIS}

Based on Lemma 2, the multi-sinusoidal disturbance $d(t)$ can be rewritten as

$$
d(t)=\frac{1}{m} \sum_{i=1}^{m}\left(\Xi_{i o} \theta_{o}+\Xi_{i e} g_{i e}+\theta_{i}^{T} \delta_{i}\right)
$$

Now that the problem can be considered in the DOBC framework, where control input $\tau$ can be constructed as

$$
\tau=M u+h_{1}(\dot{q}, q)-\hat{d}
$$

where $u \in R$ is the feedback controller to be designed. The disturbance generator gives the estimation value of disturbance $\hat{d}$ satisfying

$$
\begin{aligned}
\hat{d}(t) & =\frac{1}{m} \sum_{i=1}^{m}\left(\Xi_{i o} \hat{\theta}_{o}+\Xi_{i e} g_{i e}\right)=\frac{V_{0}}{m} \Xi_{o} \hat{\theta}_{o}+\frac{1}{m} \sum_{i=1}^{m} \Xi_{i e} g_{i e}, \\
V_{0} & =[1,1 \cdots 1], V_{0} \in R^{1 \times m}
\end{aligned}
$$

Substituting (31) into (1) yields

$$
\ddot{q}=u+\frac{V_{0}}{M m} \Xi_{o}(t) \tilde{\theta}_{o}+\frac{V_{0}}{M m} \theta^{T} \delta+\frac{1}{M} \Delta h(\dot{q}, q)
$$

Thus, the dynamic system (1) can be rewritten as

$$
\dot{x}=A x+B\left(u+\frac{1}{M} \Delta h(\dot{q}, q)+\frac{V_{0}}{M m} \Xi_{o} \tilde{\theta}_{o}+\frac{V_{0}}{M m} \theta^{T} \delta\right)
$$

where

$$
x=\left[\begin{array}{c}
q \\
\dot{q}
\end{array}\right], A=\left[\begin{array}{cc}
0 & I \\
0 & 0
\end{array}\right], B=\left[\begin{array}{l}
0 \\
I
\end{array}\right],
$$

with the feedback controller $u=K x$, where $K$ is the designed control gain for stabilization.

As we know, $\theta$ is a constant matrix relevant to disturbance frequencies $\omega_{i}(i=1,2 \ldots n)$. From Theorem 1 , if the regions of $\omega_{i}(i=1,2 \ldots n)$ are given, we can also figure out the region of $\theta$. To reduce the conservativeness, we denote $\theta_{0}$ and $\Delta \theta$ as nominal constant component and uncertain part of $\theta$ respectively, i.e.

$$
\theta=\theta_{0}+\Delta \theta
$$

Then we can find corresponding weighting matrices $U_{2}$ satisfying

$$
\left\|\Delta \theta^{T} \delta\right\| \leq\left\|U_{2} \delta\right\|
$$

The composite system including (29) and (33) yields

$$
\dot{\bar{x}}=\bar{A} x+\bar{F} \bar{f}(\bar{x}, t)
$$

where

$$
\begin{aligned}
& \bar{x}=\left[\begin{array}{c}
x \\
\tilde{\theta}_{o} \\
\delta
\end{array}\right], \bar{A}=\left[\begin{array}{ccc}
A+B K & \frac{B V_{0}}{M m} \Xi_{o} & \frac{B V_{0}}{M m} \theta_{0}^{T} \\
0 & -\alpha \Xi_{o}^{2} & -\alpha \Xi_{o} \theta_{0}^{T} \\
0 & 0 & G
\end{array}\right], \\
& \bar{F}=\left[\begin{array}{cc}
\frac{B}{M} \hat{M} & \frac{B V_{0}}{M m} \\
-\alpha \Xi_{o} \hat{L} & -\alpha \Xi_{o} \\
-L & 0
\end{array}\right], \bar{f}(\bar{x}, t)=\left[\begin{array}{c}
\Delta h(\dot{q}, q) \\
\Delta \theta^{T} \delta
\end{array}\right]
\end{aligned}
$$

Thus, according to (6) and (35) it can be derived that there exists weighting matrix $U$ satisfying $\|\bar{f}(\bar{x}, t)\|<\|U \bar{x}\|$, where

$$
U=\left[\begin{array}{ccc}
U_{1} & 0 & 0 \\
0 & 0 & U_{2}
\end{array}\right], U_{1}=\left[\begin{array}{cc}
U_{11} & 0 \\
0 & U 12
\end{array}\right]
$$

We are now in a position to find $K$ such that the close-loop system (36) with $u=K x$ is asymptotically stable. We denote $\operatorname{sym}(M):=M+M^{T}$ and present the following result.

Theorem 2: For given $\lambda>0$, if $\operatorname{Rank}\left(\bar{\Xi}_{o}(t)=m\right.$ and there exists $Q_{1}>0, Q_{3}>0$ and $R_{1}, R_{2} \in R$ satisfying

$$
\Omega=\left[\begin{array}{ccc}
\Omega_{1} & \Omega_{2} & \Omega_{3} \\
* & -I & 0 \\
* & 0 & -I
\end{array}\right]<0
$$

where

$$
\begin{aligned}
& \Omega_{1}=\left[\begin{array}{ccc}
\Omega_{11} & \frac{B V_{0}}{M m} & \frac{B V_{0}}{M m} \theta_{o}^{T} \\
* & \Omega_{12} & -R_{2} \theta_{0}^{T} \\
* * & * & \Omega_{13}
\end{array}\right], \Omega_{12}=-2 R_{2} I, \\
& \Omega_{11}=\operatorname{sym}\left(\left(A Q_{1}+B R_{1}\right)\right), \Omega_{13}=\operatorname{sym}\left(G^{T} Q_{3}\right) \\
& \Omega_{2}=\lambda\left[\begin{array}{cc}
\frac{B}{M} & \frac{B V_{0}}{M m} \\
-R_{2} \hat{L} & -R_{2} \\
-Q_{3} L & 0
\end{array}\right], \Omega_{3}=\left[\begin{array}{cc}
\frac{1}{\lambda} Q_{1} U_{1}^{T} & 0 \\
0 & 0 \\
0 & \frac{1}{\lambda} U_{2}^{T}
\end{array}\right],
\end{aligned}
$$

for arbitrary given $Q_{2}>0$ with compatible dimensions, then the composite system (36) under DOBC law (31) with $K=$ $R_{1} Q_{1}^{-1}, \alpha=R_{2} Q_{2}$ is asymptotically stable.

Proof: Let $P_{1}=Q_{1}^{-1}, P_{2}=Q_{2}^{-1}$. We construct

$$
V(\bar{x}, t)=\bar{x}^{T} P \bar{x}+\frac{1}{\lambda^{2}} \int_{0}^{t}\left(\|U \bar{x}(\epsilon)\|^{2}-\|\bar{f}(\bar{x}, \epsilon)\|^{2}\right) d \epsilon,
$$

with

$$
P=\left[\begin{array}{ccc}
P_{1} & 0 & 0 \\
0 & P_{2} I & 0 \\
0 & 0 & Q_{3}
\end{array}\right]
$$

Then

$$
\begin{aligned}
\dot{V}(\bar{x}, t)= & \bar{x}^{T}\left(P \bar{A}+\bar{A}^{T} P\right) \bar{x}+2 \bar{x}^{T} P \bar{F} \bar{f}(\bar{x}, t) \\
& +\frac{1}{\lambda^{2}}\|U \bar{x}\|^{2}-\frac{1}{\lambda^{2}}\|\bar{f}(\bar{x}, t)\|^{2} \\
= & {\left[\begin{array}{c}
\bar{x} \\
\frac{\bar{f}(\bar{x})}{\lambda}
\end{array}\right]^{T} \Lambda\left[\begin{array}{c}
\bar{x} \\
\frac{\bar{f}(\bar{x})}{\lambda}
\end{array}\right] }
\end{aligned}
$$


where

$$
\Lambda=\left[\begin{array}{cc}
\operatorname{sym}(P \bar{A})+\frac{1}{\lambda^{2}} U^{T} U & \lambda P \bar{F} \\
* & -I
\end{array}\right]
$$

Substituting (34), (35) and (38) into (43) yields

$$
\Lambda=\left[\begin{array}{cc}
\bar{\Omega}_{1} & \bar{\Omega}_{2} \\
* & -I
\end{array}\right]
$$

where

$$
\begin{aligned}
\bar{\Omega}_{1} & =\left[\begin{array}{ccc}
\bar{\Omega}_{11} & \frac{P_{1} B V_{0}}{M m} \Xi_{o} & \frac{P_{1} B V_{0}}{M m} \theta_{o}^{T} \\
* & -2 \Xi_{o}^{T} R_{2} \Xi_{o} & -R_{2} \Xi_{o} \theta_{0}^{T} \\
* & * & \operatorname{sym}\left(Q_{3} G\right)+\frac{1}{\lambda^{2}} U_{2}^{T} U_{2}
\end{array}\right], \\
\bar{\Omega}_{2} & =\lambda\left[\begin{array}{cc}
P_{1} \frac{B}{M} & P_{1} \frac{B V_{0}}{M m} \\
-R_{2} \Xi_{o} \hat{L} & -R_{2} \Xi_{o} \\
-Q_{3} L & 0
\end{array}\right], \\
\bar{\Omega}_{11} & =\operatorname{sym}\left(P_{1}(A+B K)\right)+\frac{1}{\lambda^{2}} U_{1}^{T} U_{1}
\end{aligned}
$$

Rewrite $\Lambda$ as

$$
\Lambda=\bar{\Lambda}^{T} \Lambda_{1} \bar{\Lambda}
$$

where

$$
\begin{aligned}
& \bar{\Lambda}=\left[\begin{array}{cccccc}
I & 0 & 0 & 0 & 0 & 0 \\
0 & \Xi_{o} & 0 & 0 & 0 & 0 \\
0 & 0 & I & 0 & 0 & 0 \\
0 & 0 & 0 & I & 0 & 0 \\
0 & 0 & 0 & 0 & I & 0 \\
0 & 0 & 0 & 0 & 0 & I
\end{array}\right], \Lambda_{1}=\left[\begin{array}{cc}
\overline{\bar{\Omega}}_{1} & \overline{\bar{\Omega}}_{2} \\
* & I
\end{array}\right], \\
& \overline{\bar{\Omega}}_{1}=\left[\begin{array}{ccc}
\bar{\Omega}_{11} & \frac{P_{1} B V_{0}}{M m} & \frac{P_{1} B V_{0}}{M m} \theta_{o}^{T} \\
* & -2 R_{2} & -R_{2} \theta_{0}^{T} \\
* & * & Q_{3} G+G^{T} Q_{3}+\frac{1}{\lambda^{2}} U_{2}^{T} U_{2}
\end{array}\right], \\
& \overline{\bar{\Omega}}_{2}=\lambda\left[\begin{array}{cc}
P_{1} \frac{B}{M} & P_{1} \frac{B V_{0}}{M m} \\
-R_{2} \hat{L} & -R_{2} \\
-Q_{3} L & 0
\end{array}\right]
\end{aligned}
$$

Pre-multiplying and post-multiplying simultaneously by diag $\{Q, I\}$, we can see that $\Lambda_{1}<0$ is equivalent to $\Lambda_{2}<0$, where

$$
\Lambda_{2}=\left[\begin{array}{cc}
Q \overline{\bar{\Omega}}_{1} Q & Q \overline{\bar{\Omega}}_{2} \\
* & I
\end{array}\right],
$$

and

$$
Q=\left[\begin{array}{ccc}
Q_{1} & 0 & 0 \\
0 & I & 0 \\
0 & 0 & I
\end{array}\right]
$$

Based on Schur complement, it is straightforward that $\Lambda_{2}<0$ is equivalent to $\Omega<0$. Thus we have $\dot{V}(\bar{x}, t) \leq 0$ and $\bar{x}$ is bounded. With simple calculations we further observe that $\ddot{V}(\bar{x}, t)$ is also bounded. As $G_{i}(i=1,2 \cdots m)$ is different with each other, there exists $t_{0}$ such that $\operatorname{Rank}\left(\bar{\Xi}_{o}(t)\right)<m$ for $\forall t>t_{0}$, from Barbalat lemma, $\bar{x}(t) \rightarrow 0$ as $t \rightarrow+\infty$, which completes the proof.

$$
\text { Q.E.D }
$$

Now that we can summarize the two-layer observer based control design procedure as follows:
Step 1) Select weighting matrices $G$ and $L$ in the form of (15) and (16). Then apply $G$ and $L$ in Theorem 2 , and solve (39) to obtain the feedback controller gain $K$ and $\alpha$.

Step 2) Design the auxiliary observer for $\xi$ according to (13) such that (24) is satisfied. Construct the augmented observer based on (27) to estimate unknown constant vector $\theta_{o}$. Then the two-layer disturbance observer $\hat{d}$ can be derived as (32).

Step 3) Apply $\hat{d}$ and the feedback controller $u=K x$ to (31), and obtain the DOBC controller $\tau$.

Remark 1: The convergence speed depends not only on the design of $\alpha$, but also the selection of $G$ because it determines the value of $\theta_{o}$. From Equ.(12) we can get the correlation between $G$, frequencies and $\theta_{o}(0)$. If we have a priori information on the range of the disturbance frequencies, a reasonable selection of $z(0), p(0)$ and $G$ can help reducing large initial values of $\tilde{\theta}_{o}, \delta$, and achieving a faster convergence property.

\section{SIMULATIONS}

The proposed control architecture will be deployed to a numerical example of the single-link robotic manipulator model (1) to verify its effectiveness. In particular, the gravity term is expressed as $G(q)=m g l \cos (q)$, and the parameters in (1) are given by $m_{0}=1, l=0.25, V_{m}=2.0, g=9.8, M=3 / 4 m_{0} l^{2}$. With this, the control law in (31) can be applied, and the corresponding parameters in (33) can be obtained

$$
A=\left[\begin{array}{ll}
0 & 1 \\
0 & 0
\end{array}\right], B=\left[\begin{array}{l}
0 \\
1
\end{array}\right]
$$

Suppose that the uncertainty in $h(q, \dot{q})=V_{m} \dot{q}+G(q)$ is described by parameter perturbations satisfying

$$
\|\Delta h(\dot{q}, q)\| \leq\left\|\left[\begin{array}{ll}
2 & 0 \\
0 & 2
\end{array}\right]\left[\begin{array}{c}
q \\
\dot{q}
\end{array}\right]\right\| .
$$

With definition of (36), $U_{i}(i=1,2)$ in (38) can be set as $U_{1}=$ $\operatorname{diag}\{2,2\}, U_{2}=\operatorname{diag}\{5000,0,5000,0,5000,0,5000,0\}$

We would like to conduct various simulations to demonstrate the effectiveness of the proposed algorithm. In particular, we are interested in the anti-disturbance control performance for the case of low frequency disturbances, as well as the case of high frequency disturbances. In the meanwhile, we would like to investigate the control performance with the existence of system uncertainties.

Case I. $d=\sin (t)+\sin (4 t)$

As a first example, we consider the situation of lowfrequency disturbance rejection. $G_{1,2}$ and $L_{1,2}$ in (10) are chosen 
as

$$
\begin{aligned}
G_{1} & =\left[\begin{array}{llll}
0 & 1 & 0 & 0 \\
0 & 0 & 1 & 0 \\
0 & 0 & 0 & 1 \\
-16 & -32 & -24 & -8
\end{array}\right], \\
G_{2} & =\left[\begin{array}{llll}
0 & 1 & 0 & 0 \\
0 & 0 & 1 & 0 \\
0 & 0 & 0 & 1 \\
-16 & -33 & -24 & -9
\end{array}\right], \\
L_{1,2} & =\left[\begin{array}{llll}
0 & 0 & 0 & 1
\end{array}\right]^{T}
\end{aligned}
$$

Based on Theorem 2 we have

$$
K=\left[\begin{array}{ll}
-281.4448 & -120.4225
\end{array}\right], \alpha=5000 .
$$

As depicted in Fig.2, the proposed disturbance observer can well capture the harmonic disturbances, and the estimation error converges to zero fast. As a result, the motion control precision of the robotic manipulator can be guaranteed by robust feedback controller. Note that the augmented observer gives the estimation value of parameters related to disturbance frequencies as $\hat{\theta}_{o}(1)=0, \hat{\theta}_{o}(2)=7$. Recall $(12)$, we know that the multi-sinusoidal disturbance frequencies $\omega_{1}$ and $\omega_{2}$ should satisfy $\omega_{1}^{2} \omega_{2}^{2}=16-\theta_{o}(1), \omega_{1}^{2}+\omega_{2}^{2}=24-\theta_{o}(2)$, which can be illustrated in Fig.2 as well.

Case II. $d=\sin (t)+\sin (20 t)$

High frequency disturbance estimation and rejection is usually more challenging. In this example, we introduce a higher frequency component in the disturbance. Note that the proposed method has more flexibility in parameter selections of $G$ and $L$, which can benefit convergence performance. In this example, $G_{1,2}$ and $L_{1,2}$ in (10) are chosen as

$$
\begin{aligned}
G_{1}= & {\left[\begin{array}{llll}
0 & 1 & 0 & 0 \\
0 & 0 & 1 & 0 \\
0 & 0 & 0 & 1 \\
-120 & -154 & -71 & -14
\end{array}\right], } \\
G_{2}= & {\left[\begin{array}{lllll}
0 & 1 & 0 & 0 & \\
0 & 0 & 1 & 0 & \\
0 & 0 & 0 & 1 \\
-120 & -160 & -71 & -15
\end{array}\right], } \\
L_{1,2}= & {\left[\begin{array}{llllllll}
0 & 0 & 0 & 1 & 0 & 0 & 0 & 1
\end{array}\right]^{T} }
\end{aligned}
$$

Meanwhile, we pick up $\alpha=50000$ and solve (39) to get the feedback gain as

$$
K=\left[\begin{array}{ll}
-128.3 & -22.7
\end{array}\right]
$$

As depicted in Fig.3, the disturbance estimation error and system output can still converges with good transient responses. Meanwhile, $\hat{\theta}_{o}$ can track $\theta_{o}$ very fast even for higher disturbance frequencies, which means that the unknown frequencies of the disturbance can be rapidly estimated.

Case III. $d=\sin (t)+\sin (20 t)$ with system uncertainties

To test the robustness of the proposed method against system uncertainties, we consider the case with the parameter perturbation in viscous friction coefficient $V_{m}$. Similar to Case II, we test the high frequency disturbance rejection, but with the deviation of the true value of $V_{m}=3$. As depicted in Fig.4, the convergence speed of estimation error and system output are slower and their transient responses are more oscillatory, due to the existence of parameter perturbations. However the algorithm can still guarantee system stability asymptotically. Meanwhile it is still possible to further improve its performance with different selections of controller gain $K$ and the free parameter $\alpha$. asymptotically stability.

Another case with disturbances of three frequency components is also conducted to further verify the proposed strategy. In this particular case, a larger parametric perturbation is also added with $V m=4$ ). The following parameters have been adopted for the simulations. As depicted in Fig.5, the proposed disturbance observer can well capture the multiple frequency components of the disturbance, with fast convergence of estimations.

$$
\begin{aligned}
& G_{1}=\left[\begin{array}{llllll}
0 & 1 & 0 & 0 & 0 & 0 \\
0 & 0 & 1 & 0 & 0 & 0 \\
0 & 0 & 0 & 1 & 0 & 0 \\
0 & 0 & 0 & 0 & 1 & 0 \\
0 & 0 & 0 & 0 & 0 & 1 \\
-64 & -192 & -240 & -160 & -60 & -12
\end{array}\right], \\
& G_{2}=\left[\begin{array}{llllll}
0 & 1 & 0 & 0 & 0 & 0 \\
0 & 0 & 1 & 0 & 0 & 0 \\
0 & 0 & 0 & 1 & 0 & 0 \\
0 & 0 & 0 & 0 & 1 & 0 \\
0 & 0 & 0 & 0 & 0 & 1 \\
-64 & -190 & -240 & -170 & -60 & -10
\end{array}\right] \text {, } \\
& G_{3}=\left[\begin{array}{llllll}
0 & 1 & 0 & 0 & 0 & 0 \\
0 & 0 & 1 & 0 & 0 & 0 \\
0 & 0 & 0 & 1 & 0 & 0 \\
0 & 0 & 0 & 0 & 1 & 0 \\
0 & 0 & 0 & 0 & 0 & 1 \\
-64 & -195 & -240 & -165 & -60 & -152
\end{array}\right] \\
& L=\left[\begin{array}{lll}
L_{1} & L_{2} & L_{3}
\end{array}\right]^{T} \text {, } \\
& L_{i}=\left[\begin{array}{llllll}
0 & 0 & 0 & 0 & 0 & 1
\end{array}\right],(i=1,2,3) \\
& K=\left[\begin{array}{ll}
-155.5 & -81.4
\end{array}\right] \text {. }
\end{aligned}
$$

Case IV. Comparison with the typical DOBC method

In typical DOBC schemes, disturbance key parameter$s$ must be available. Otherwise the uncertainty part will have to be treated as perturbations to be attenuated. In the following simulation, the actual disturbance equals to $\sin (t)+\sin (20 t)$ for the first 10 seconds, and then changes to $\sin (t)+\sin (15 t)$. For comparison purpose, we use traditional DOBC method (see $[9,10]$ ) with fixed frequency values by letting $\omega_{1}=1, \omega_{2}=19$. It is noted that the control precision will deteriorate significantly with traditional DOBC scheme, while the proposed two-layer observer based control achieves much better tracking performance due to its capability to capture the disturbance key parameters such as frequency values, which is depicted in Fig, 6. 

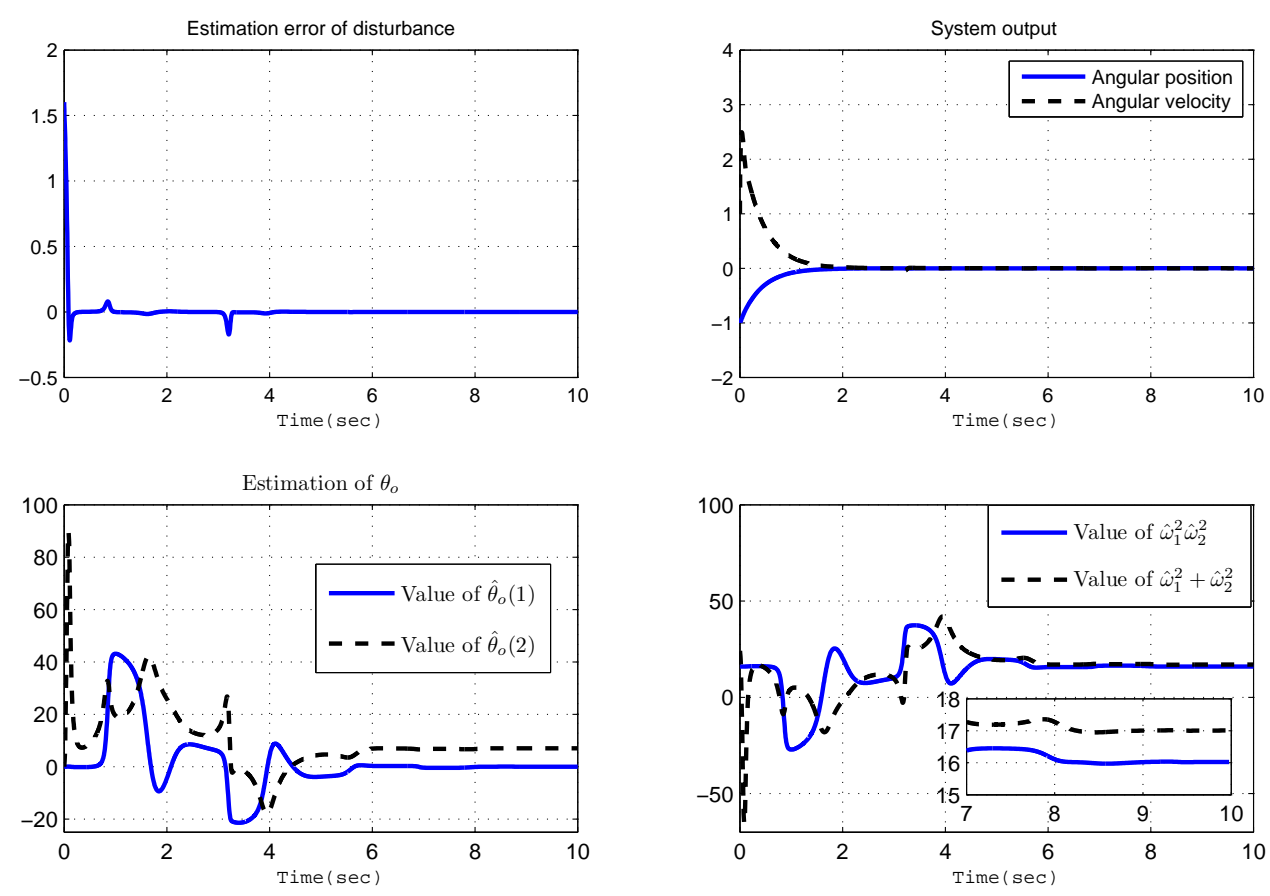

Figure 2: System performance using proposed algorithm $(d=\sin (t)+\sin (4 t))$
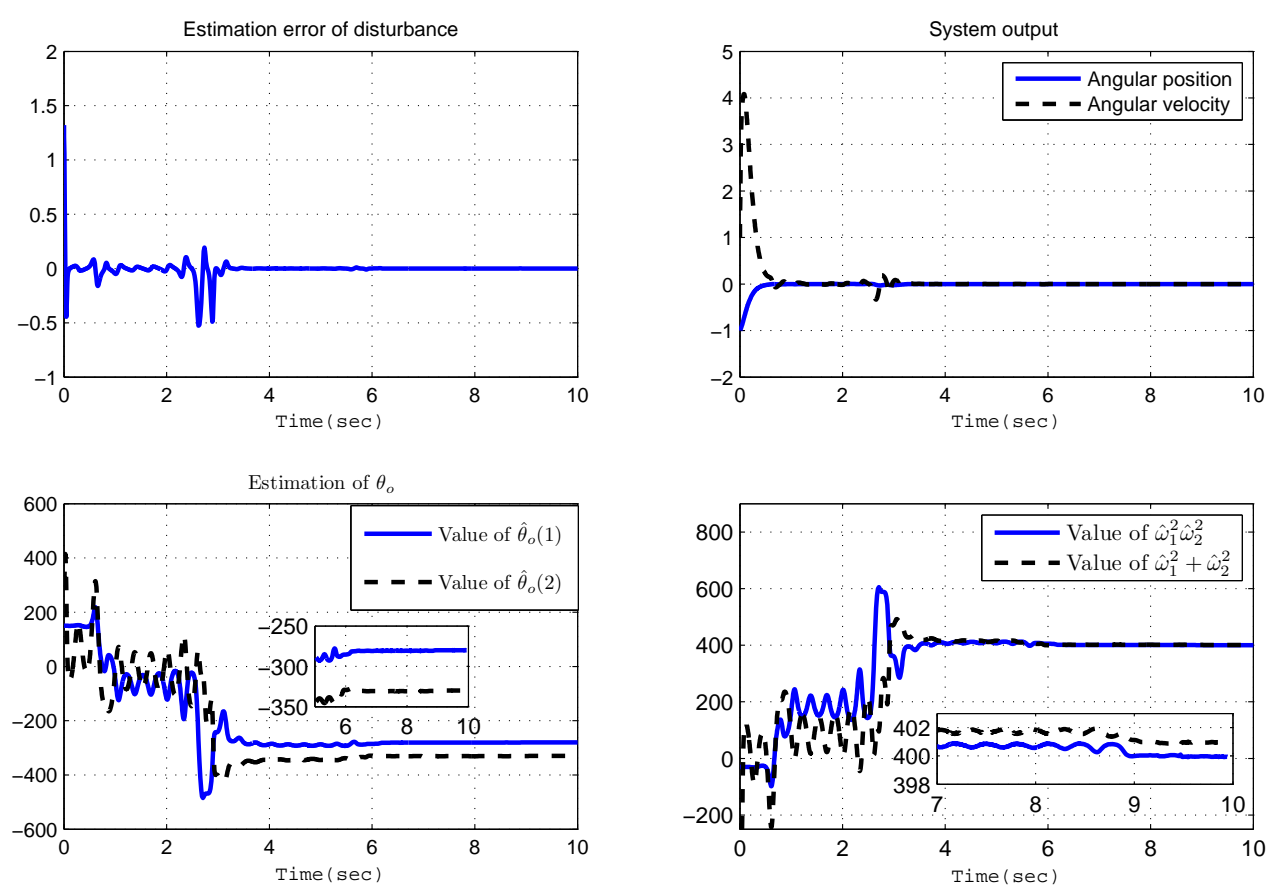

Figure 3: System performance using proposed algorithm $(d=\sin (t)+\sin (20 t))$

\section{Conclusion}

Anti-disturbance control for dynamical uncertain systems subject to disturbances with multiple unknown-frequency com- ponents was considered in this paper, where a novel two-layer disturbance observer based control architecture was developed. With the augmented observer structure, the control problem can be converted to a DOBC problem where asymptotic stability 

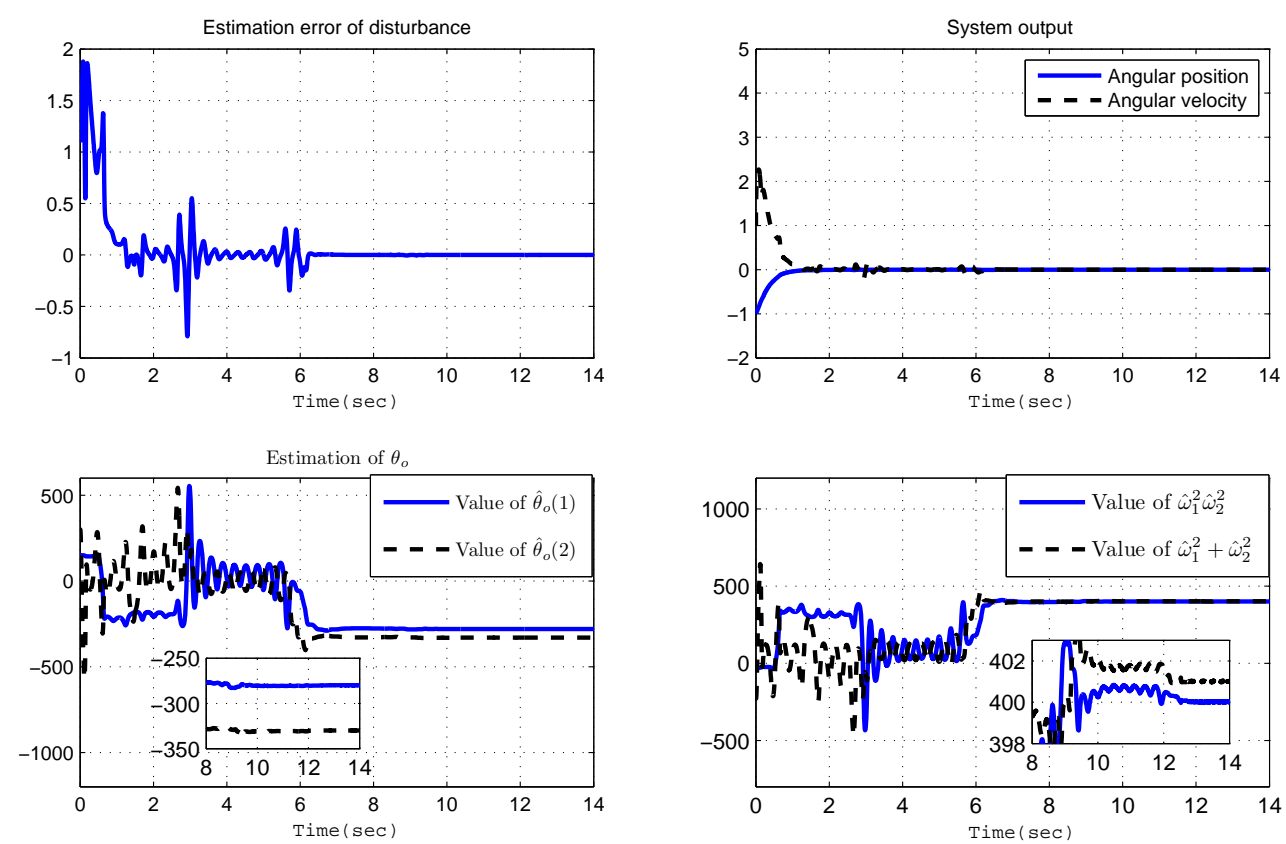

Figure 4: System performance using proposed algorithm with parameter perturbation $(d=\sin (t)+\sin (20 t))$
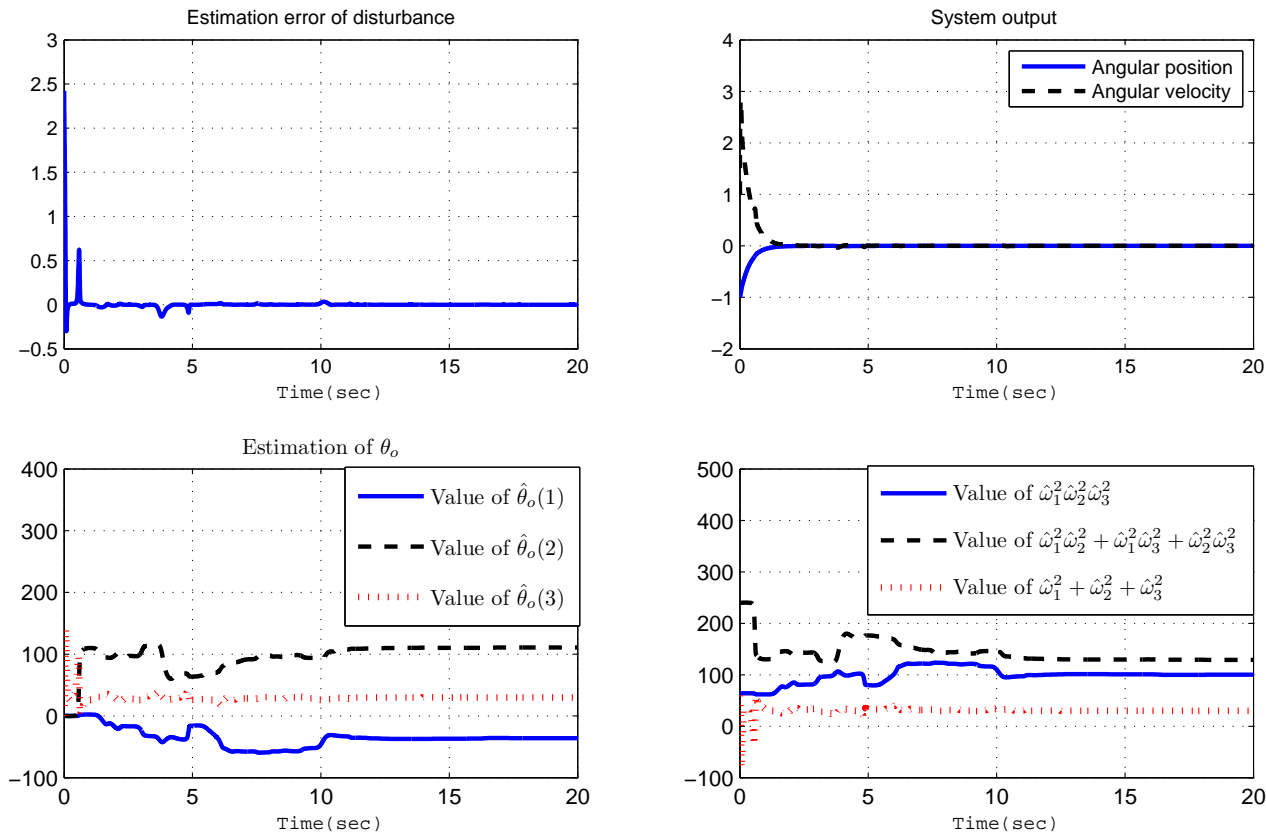

Figure 5: System performance using proposed algorithm with parameter perturbation $(d=\sin (t)+\sin (2 t)+\sin (5 t))$

can be guaranteed with robust stabilizer design. The proposed approach was successfully validated on a robotic manipulating example, where low frequency and high frequency disturbances can be well handled even in the presence of system parameter perturbations.

\section{References}

[1] Chen X K, Su C Y, Toshio Fukuda. A Nonlinear Disturbance Observer for Multivariable Systems and Its Application to Magnetic Bearing Systems. IEEE Transactions Control Systems Technology 2004;12(4):569-577.

[2] She J, Ohyama Y,Nakano M. A New Approach to the Estimation and Rejection of Disturbances in Servo Systems. IEEE Transactions on Control Systems Technology 2005;13(3):378-385. 

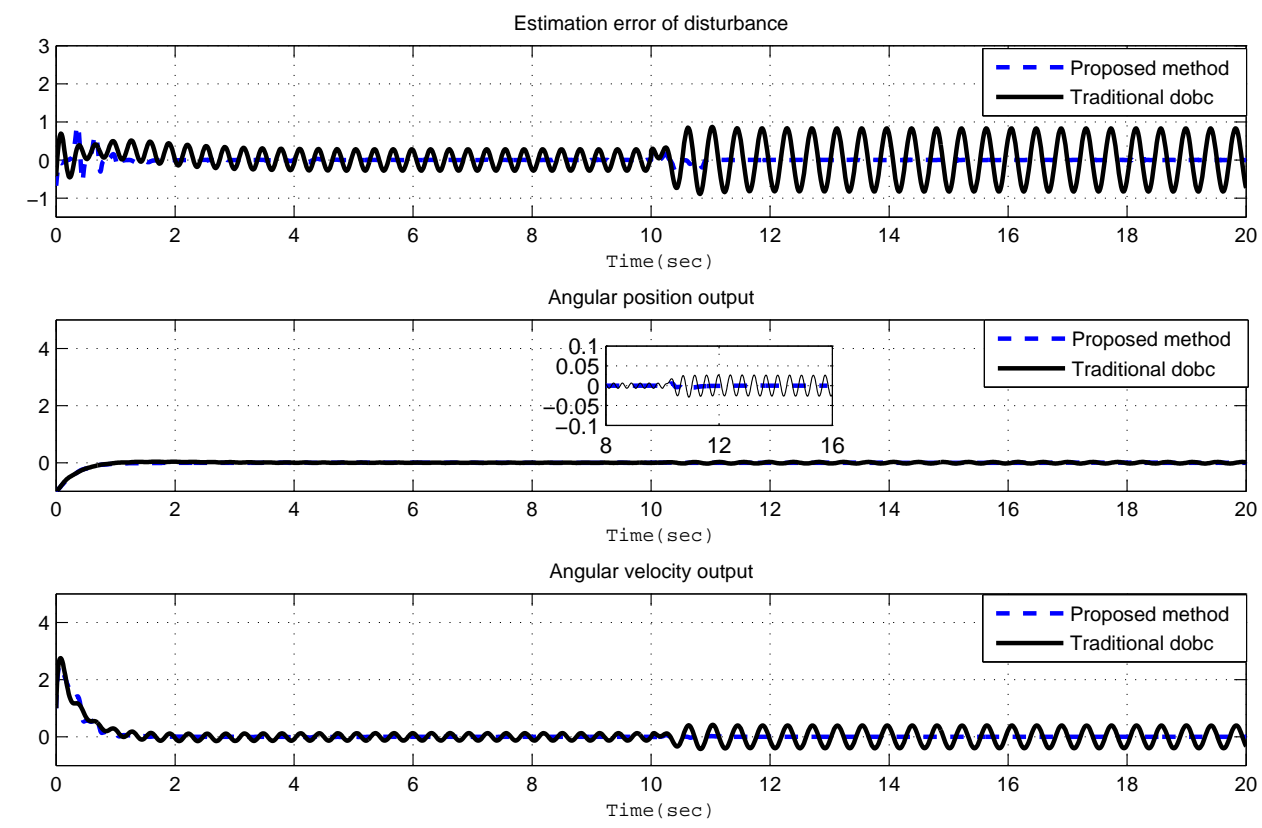

Figure 6: Comparison of system performance using two-layer observer based control and traditional DOBC

[3] Chen W H. Disturbance Observer Based Control for Nonlinear Systems. IEEE/ASME Transactions on Mechatronics 2004;9(4):706-710.

[4] Qing W J. Disturbance Rejection Through Disturbance Observer With Adaptive Frequency Estimation. IEEE Transactions on Magnetics 2009;45(6): 2675-2678.

[5] Back J, Shim H.Adding robustness to nominal output-feedback controllers for uncertain nonlinear systems: A nonlinear version of disturbance observer.Automatica 2008; 44(9):2528-2537.

[6] Yang J, Li S H.Robust autopilot design for bank-to-turn missiles using disturbance observers.IEEE Transactions on Aerospace and Electronic Systems 2013; 49(1): 558-579.

[7] Yang J, Li S H.Sliding-Mode Control for Systems With Mismatched Uncertainties via a Disturbance Observer.IEEE Transactions on Industrial Electronics 2013;60(1): 160-169.

[8] Guo L,Chen W H.Disturbance attenuation and rejection for systems with nonlinearity via DOBC approach.International Journal of Robust Nonlinear Control 2005;15(3): 109-125.

[9] Wei X, Guo L.Composite Disturbance-Observer-Based Control and $H_{\infty}$ Control for Complex Continuous Models.International Journal of Robust and nonlinear Control 2010; 20(1); 106-118.

[10] Wen X Y,Guo L, Yan P.Composite hierarchical anti- disturbance control for robotic systems with multiple disturbances.International Journal of Control Automation and Systems 2014; 12(3): 541-551.

[11] Guo L, Wen X Y.Hierarchical anti-disturbance adaptive control for nonlinear systems with composite disturbances and applications to missile systems.Transaction of the Institute of Measurement and Control 2011;33(8):942-956.

[12] Chen W H. A Nonlinear Disturbance Observer for Robotic Manipulators.IEEE Transactions on Industrial Electronics 2000; 47(4):932-938.

[13] Li S H, Yang J, Chen W H, Chen X S. Disturbance Observer Based Control: Methods and Applications 2014. CRC Press Inc.

[14] Chen W H, Yang J, Guo L, Li S H. Disturbance observer-based control and related methods: An overview. IEEE Transactions on Industrial Electronics, Published online.
[15] Yang J, Zheng W X, Li S H. Design of a prediction accuracy enhanced continuous-time MPC for disturbed systems via a disturbance observer. IEEE Transactions on Industrial Electronics 2015; 62(9):5807-5816.

[16] Guo L, Cao S Y. Anti-disturbance control theory for systems with multiple disturbances: A survey. ISA Transactions 2014; 53(4):846849.

[17] Bodson M,Jensen J S, DouglasS C. Active noise control for periodic disturbances.IEEE Transactions on Control Systems Technology 2001; 9(1): 200-205.

[18] Pigg S, Bodson M. Adaptive algorithms for the rejection of sinusoidal disturbances acting on unknown plants. IEEE Transactions on Control System Technology 2010;18(4), 822-836.

[19] Nikiforov V.Nonlinear servocompensation of unknown external disturbances. Automatica 2001; 37(10): 1647-1653.

[20] Maurizio F, Foued B A.Adaptive regulation of MIMO linear systems against unknown sinusoidal exogenous inputs. International Journal of Adaptive Control and Signal Processing 2009; 23(6): 581-603.

[21] Marino R, Tomei P.Disturbance cancellation for linear systems by adaptive internal models. Automatica 2013;49(5):1494-1500.

[22] Serrani A, Isidori A, Marconi L.Semiglobal nonlinear output regulation with adaptive internal model. IEEE Transactions on Automatic Control 2001; 46(8):1178-1194.

[23] Ding Z.Global stabilization and disturbance suppression of a class of nonlinear systems with uncertain internal model. Automatica 2003;39(3):471-479.

[24] Ding Z.Universal disturbance rejection for nonlinear systems in output feedback form.IEEE Transactions on Automatic Control 2003; 48(7): $1222-1226$.

[25] Xi Z R, Ding Z.Global adaptive output regulation of a class of nonlinear systems with nonlinear exosystems.Automatica 2007; 43(1): 143-149.

[26] Wen X Y, Guo L. Estimation and Rejection of Unknown Sinusoidal Disturbances Using TSNLDO. Anti-Disturbance Control for Systems with Multiple Disturbances 2013; CRC Press Inc. 